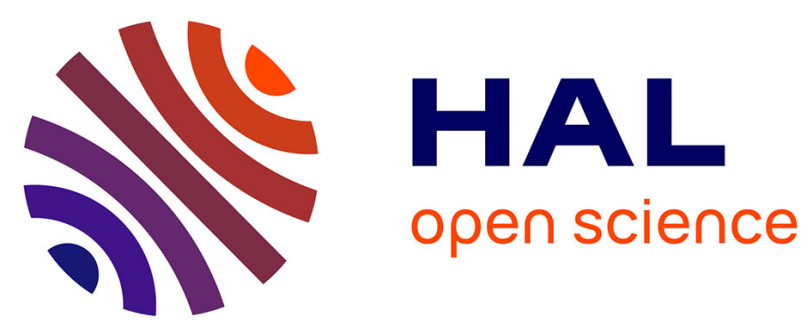

\title{
Automated Setup of Multi-Cloud Environments for Microservices Applications
}

\author{
Gustavo Sousa, Walter Rudametkin, Laurence Duchien
}

\section{To cite this version:}

Gustavo Sousa, Walter Rudametkin, Laurence Duchien. Automated Setup of Multi-Cloud Environments for Microservices Applications. 2016 IEEE 9th International Conference on Cloud Computing (CLOUD'16), Jun 2016, San Francisco, United States. pp.327-334, 10.1109/CLOUD.2016.0051 . hal01312606v2

\section{HAL Id: hal-01312606 https://hal.inria.fr/hal-01312606v2}

Submitted on 7 Mar 2017

HAL is a multi-disciplinary open access archive for the deposit and dissemination of scientific research documents, whether they are published or not. The documents may come from teaching and research institutions in France or abroad, or from public or private research centers.
L'archive ouverte pluridisciplinaire HAL, est destinée au dépôt et à la diffusion de documents scientifiques de niveau recherche, publiés ou non, émanant des établissements d'enseignement et de recherche français ou étrangers, des laboratoires publics ou privés. 


\title{
Automated Setup of Multi-Cloud Environments for Microservices Applications
}

\author{
Gustavo Sousa, Walter Rudametkin, Laurence Duchien \\ Université de Lille / CRIStAL UMR 9189 / École Centrale de Lille \\ Inria \\ Lille, France \\ Email: firstname.lastname@inria.fr
}

\begin{abstract}
Multi-cloud computing has been proposed as a way to reduce vendor dependence, comply with location regulations, and optimize reliability, performance and costs. Meanwhile, microservice architectures are becoming increasingly popular in cloud computing as they promote decomposing applications into small services that can be independently deployed and scaled, thus optimizing resources usage.

However, setting up a multi-cloud environment to deploy a microservices-based application is still a very complex and time consuming task. Each microservice may require different functionality (e.g. software platforms, databases, monitoring and scalability tools) and have different location and redundancy requirements. Selection of cloud providers should take into account the individual requirements of each service, as well as the global requirements of reliability and scalability. Moreover, cloud providers can be very heterogeneous and offer disparate functionality, thus hindering comparison.

In this paper we propose an automated approach for the selection and configuration of cloud providers for multi-cloud microservices-based applications. Our approach uses a domain specific language to describe the application's multi-cloud requirements and we provide a systematic method for obtaining proper configurations that comply with the application's requirements and the cloud providers' constraints.
\end{abstract}

Index Terms-multi-cloud; microservices; cloud management; variability management; software product lines

\section{INTRODUCTION}

Multi-cloud computing is the use of resources and services from multiple cloud providers where there is no agreement between providers to offer an integrated view of the system [1]. It is up to the customer or a third-party to integrate services from different providers. Multi-cloud computing is a way to avoid vendor dependence and to better exploit offerings in the cloud market by employing a combination of public and private cloud resources. Customers can build configurations that better fit their needs while reducing their dependence on any given provider.

The microservices architectural style [2] provides an approach for building scalable applications. In a microservices architecture, applications are composed of small services that run in separate processes and can be deployed to different environments. Besides the benefits from separating concerns and of modularization, this approach scales services independently, allowing better use of resources.

We argue that these two approaches have strong synergism. On the one hand, building a multi-cloud application in- volves deploying to different clouds. Having independent wellisolated services facilitates this. On the other hand running microservices across private and public clouds from different providers allows for improved scalability and reliability.

However, building a multi-cloud solution is complex. Customers must consider factors such as functional requirements, configuration options, pricing policy, data center location, availability levels, etc. Cloud providers have different management interfaces and use different concepts for their offerings, making them difficult to compare. In addition, each of the application's services may have different requirements. They may be written in different programming languages, using different application frameworks or databases, and may have different scalability and availability requirements. Thus, a cloud provider that supports one of the application's services might not support another. Also, local regulations may require data to be stored within a given region, imposing further constraints.

We propose an automated approach for the selection and configuration of cloud providers for multi-cloud microservices-based applications. Our approach relies on ontology reasoning [3] and software product line techniques [4] to get from a high-level description of multi-cloud requirements to a configuration for a multi-cloud environment. While some approaches deal with aspects of multi-cloud management [5], [6], [7], [8], [9], they do not take into account the rich variability that exists in cloud providers' configuration options, losing valuable insight. Work done to manage variability in the cloud [10] does not consider multi-cloud requirements. Our approach goes further than existing work by dealing with multi-cloud requirements for microservicesbased applications, while considering provider configuration variability.

Our key contributions are:

- An approach to build multi-cloud environments that handles the intrinsic variability in existing providers.

- A method for translating high-level multi-cloud requirements to a set of provider specific feature model configurations.

- Domain specific languages for specifying an application's multi-cloud requirements.

- An experimental assessment of the approach using an example application and four popular cloud providers. 
In Section II] we discuss the motivation for multi-cloud computing and issues identified when setting up multi-cloud environments. In Section III we present our approach, and in Section IV] we discuss its implementation. Finally, we discuss related work in Section $\mathrm{V}$ and conclusions in Section $\mathrm{VI}$.

\section{Motivation}

Motivations for using multiple clouds have been discussed in recent research [11], [12]. A list of 10 reasons were identified in a survey conducted by Petcu [13]. We classify these motivations into three broad categories:

- Reducing vendor dependence. This includes protecting applications from cloud outages or failures, but also avoiding vendor lock-in.

- Optimizing performance and costs. Different cloud providers offer specific advantages. Combining them can improve QoS or costs. Multi-clouds can also be used to offload processing to other clouds when dealing with peaks, or to deploy services closer to the end user.

- Complying with requirements, constraints or regulations. In case no cloud satisfies all of the applications requirements, combining clouds becomes mandatory. Also, regulations may require sensitive data to be stored in a particular location, such as the user's country.

Multi-cloud applications exist for different reasons and approaches to manage them should take this into account.

To illustrate the complexity faced when building a multicloud system, we introduce an example scenario of an ecommerce application that requires the use of multiple clouds. This application is composed of the following services:

- a product catalog that keeps a record of product information and inventory;

- a recommendation service that records purchases and recommends new products for customers;

- a user management service that manages a user database and a credit card database;

- an order management service that tracks orders and their statuses;

- a payment service responsible for interacting with banking and credit card partners;

- as well as a web frontend and a mobile API gateway to provide access to end users' devices.

Each of these services may be developed independently, using different technologies. Therefore, each service has different functional requirements. For instance, while the web frontend is a web application written in Java, the recommendation service is a standalone Python application with an embedded database. Moreover, according to its development process, a service may also require extra tools for continuous integration, issue tracking, staging resources, etc.

In addition to functional requirements, this example scenario should also meet the following:

- instances of web frontend and product catalog should be deployed to at least two different providers to ensure essential services are always available, even in the case of a provider failure.

- data concerning European customers should be stored in a country that is a member of the European Union.

- the credit card database should be kept in a private cloud controlled by the company.

Apart from these requirements, there are no further restrictions on application services. Thus, when setting up a multicloud environment to deploy this application, we will be looking to optimize costs or improve the quality of service while complying with application requirements and constraints.

Even from this simple example we can see that taking all relevant factors into account for setting up a multi-cloud environment can be a very complex task, which calls for automation and supporting tools.

In this paper, we present an automated approach for the selection of cloud providers and generation of proper configurations that considers the following concerns:

Cloud providers heterogeneity: Cloud providers usually offer functionality as services at different levels of abstraction such as infrastructure (IaaS), platform (PaaS) and software (SaaS). Even at the same abstraction level, providers employ varying terminology and features which usually do not map directly those of competing providers. These differences complicate further the comparison between providers.

Cloud providers variability: Cloud providers may have complex rules concerning their configuration. According to the provider, some functionality may only be available in a given region or for a given user plan. Some features may have conflicts while other may have dependencies. These as well as other complex constraints can be found in cloud providers and should all be considered to obtain a proper configuration.

Multi-cloud requirements for microservices-based applications: Microservices can be developed by different teams, relying on different technologies and methodologies and may therefore require functionalities at different levels of abstraction. In a multi-cloud environment, microservices can be deployed across private and public clouds from different providers to implement scalability and redundancy mechanisms or to comply with location constraints.

\section{APPROACH}

Our approach to deal with the concerns identified in Section II relies on (i) a high-level domain-specific language for describing multi-cloud requirements; (ii) feature models to manage the variability in cloud providers; and (iii) ontologies for dealing with the heterogeneity across cloud providers.

Our approach, depicted in Fig. 1, makes a distinction between the roles of cloud provider experts and developers. A cloud provider expert is responsible for providing formal descriptions of cloud providers' offerings through feature models and ontology mappings. On the other hand, developers interact with the approach by providing requirements of a multicloud environment. These requirements are matched against providers' descriptions to generate a multi-cloud configuration, 


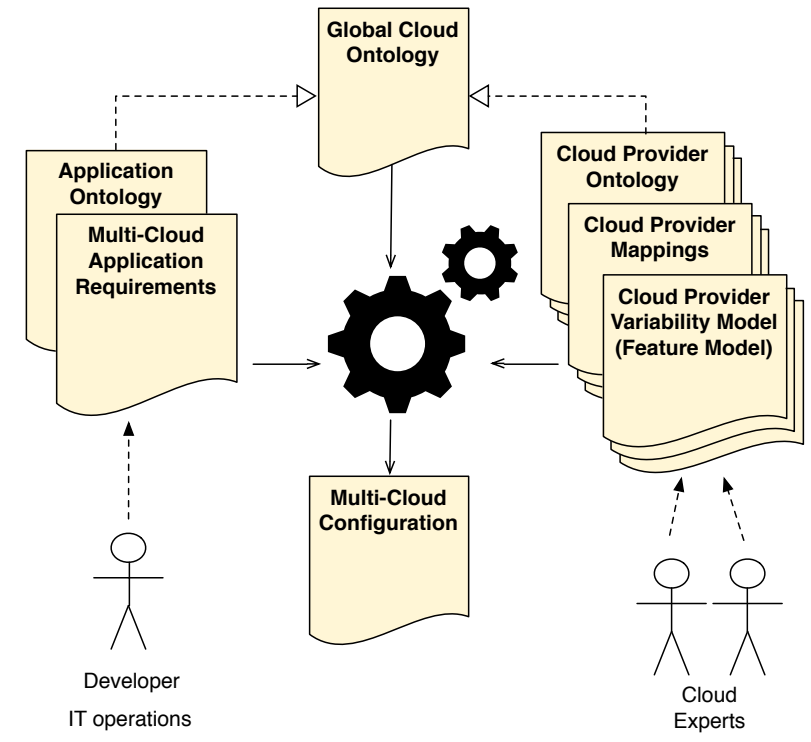

Fig. 1. Overview of the actors and artifacts involved in the proposed approach

which includes a selection of clouds providers and information on how to configure them for deploying the application.

\section{A. A DSL for multi-cloud application requirements}

We propose a language for defining multi-cloud requirements based on three main constructs: service requirements, cloud variables and instance groups. The service requirements are intended to describe the functionality required by a service to run. These can be virtual machines, runtime environments, databases, software management tools, or any other feature that is offered by a cloud provider. Fig. 2 depicts part of the requirements of the example given in Section III. For instance, the web frontend service requires a webcontainer with at least 2 GB of RAM that has a JavaWebContainer that implements version 2.5 of the Servlet API.

The concepts mentioned in service requirements are part of a global cloud ontology. If a concept is not in the ontology, the developer can use the application specific ontology to include new concepts. For example, one could add a new ontology class called HighPerformanceWebContainer and express that it is equivalent to a WebContainer that has more than 4 virtual CPUs. Once a new concept is added it can be used as part of a service requirements description.

The cloud variables describe conditions on the clouds where the services are to be deployed. This allows specifying requirements, such as, instances of a service should be placed in clouds maintained by different providers, or, that a given service should be deployed to a cloud located in a specific region. In Fig. 2, we have a condition that cloud $B$ should be located in westernEurope and that cloud $A$ should be a different provider than $B$.

Finally, instance groups describe where to deploy services and how they will be instantiated. This construct lets developers specify constraints on the number of instances at the cloud or global levels. In our example, the web frontend service will

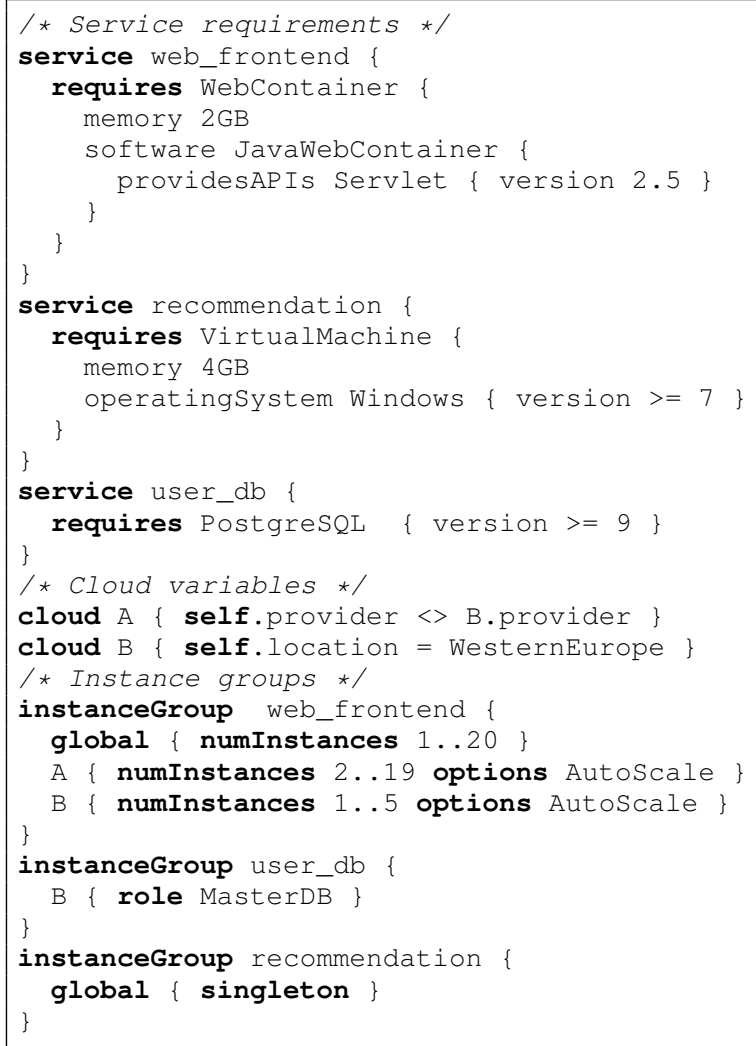

Fig. 2. Sample multi-cloud requirements

have instances running in both clouds $A$ and $B$; the user $d b$ database will be located in cloud $B$; and the recommendation service will have a singleton instance running in any cloud.

In essence, the proposed language enables developers to define requirements for each service individually, relying on concepts from the cloud ontology. The ontologies are extensible and allow defining complex requirements involving logical operators and composition rules. Finally, cloud variables and instance groups can be used to define scalability and redundancy rules across providers or regions, as well as location and colocation rules for services.

\section{B. Feature models for managing cloud variability}

Feature models are widely used to model commonalities and variability across a software product line or system family [14]. A feature model is usually depicted as a tree diagram whose nodes represent features that can be selected to build a software product. While the tree hierarchy describes a composition relationship between features, additional constraints allow for defining extra relationships between features.

In our approach, feature models are used to model the variability in the configurations of a given cloud provider. This means that a feature model describes what are the services and resources offered by a given provider, how they are related and what are the constraints between them.

Fig. 3 depicts part of a feature model elaborated to describe the variability in the OpenShift PaaS provider. OpenShift 


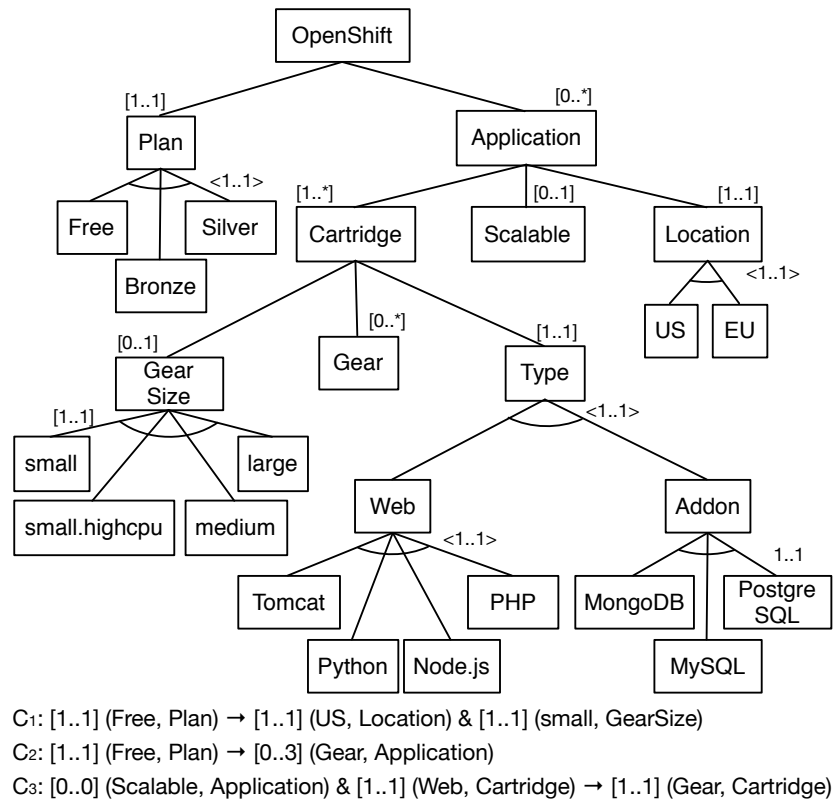

Fig. 3. Feature model for OpenShift PaaS

allows for configuring multiple Applications, which are composed by one or more Cartridges. In the OpenShift PaaS, a Cartridge defines an executable container, which can be either of type Web or Addon and can have many Gears (the running instances of the container) of a selected Gear Size. This feature model also describes additional constraints such as $C_{1}$, expressing that when the Free Plan is chosen all applications should be deployed in the US and use only small size Gears.

The feature models employed in our approach, as the one shown, are extended with cardinalities and additional constraints. Therefore, we rely on previous developments done on cardinality-based feature models [15] and on modeling cloud providers with feature models [16]. For expressing relative cardinalities and the complex constraints found in cloud providers, we also rely on the results of our previous work on extending feature models with relative cardinalities [17].

A relative cardinality is the number of instances of given feature in relation to one of its ancestors in the feature model tree hierarchy. For example, in constraint $C_{2}$ of Fig. 3. [0..3] (Gear, Application) is the cardinality of feature Gear in relation to Application in the case of feature Free being selected. This means that the maximum number of Gears in an Application is three when the Free plan is chosen.

Feature models have already been used in [18], [10] to manage the variability in a cloud provider's configuration. However, in their approach the feature models employed consider only configurations for single service applications. For instance, in the case of the OpenShift PaaS, it allows for defining a configuration for either a Java or a Python application, but it does not allow defining a configuration for an application composed of two services, written in different languages or deployed to different regions.
This limitation exists because their implementation of feature models does not consider that individual instances of a feature can have a different decomposition of its subfeatures. But it is also due to the limitation of existing feature modeling constructs, which could not describe the complex constraints involving relative cardinalities that surface once we consider configuring multiple services through feature models.

In a summary, by modeling cloud provider variability we guarantee that the configurations generated by our approach are valid according to providers' constraints. In addition, the use of feature models extended with relative cardinalities allows for modeling the complex constraints identified when service-based applications are considered.

\section{Ontologies for managing cloud heterogeneity}

Feature models can capture the variability in a cloud provider but they cannot describe the semantics of these features. In a feature model we can specify that choosing a Free plan implies using only small size Gears, but we can not describe what is a small size Gear. For describing semantics of features we rely on the use of ontologies and mappings from features to ontology concepts. An ontology is a formal definition of concepts in a given domain and how they are interrelated. It is usually defined by a set of classes and objects that are related through properties.

In our approach, mapping a feature model to an ontology is done through a domain-specific language. Fig. 4 shows part of the mapping from the OpenShift PaaS feature model to the cloud ontology. In this example, we can see a mapping from feature Tomcat to ontology class JBossEWS. This mapping states that an instance of feature Tomcat represents an object of the ontology class JBossEWS. The concepts referenced in the mapping are also part of a global cloud ontology that includes the main concepts of the cloud computing domain.

However, while in some cases a feature may provide an instance of a cloud concept by itself, in other cases a set of features may be needed. For example, the Cartridge feature in the OpenShift PaaS (Fig. 3) represents an executable container that can be used to run a web application server or a database. In the cloud ontology, executable containers are represented by the class AppContainer, which has a set of properties such as CPU power, available RAM, disk size, the kind of software it can run, in which cloud it is deployed, etc. The properties of a given instance of feature Cartridge depend on how its subfeatures are selected. Therefore, an instance of feature Cartridge will have 1GB of RAM if it is of medium size, it will provide a JBoss EWS 2.0 as a servlet container if Tomcat feature is selected, and it will be deployed to Amazon US-East-1 cloud if the US feature is selected.

To deal with this scenario the mapping language allows describing properties that depend on the selection of features. The mapping for feature Cartridge in Fig. 44illustrates how this can be done. In this case, the mappings work as a template for an ontology object, whose property values depend on the selection of features. 


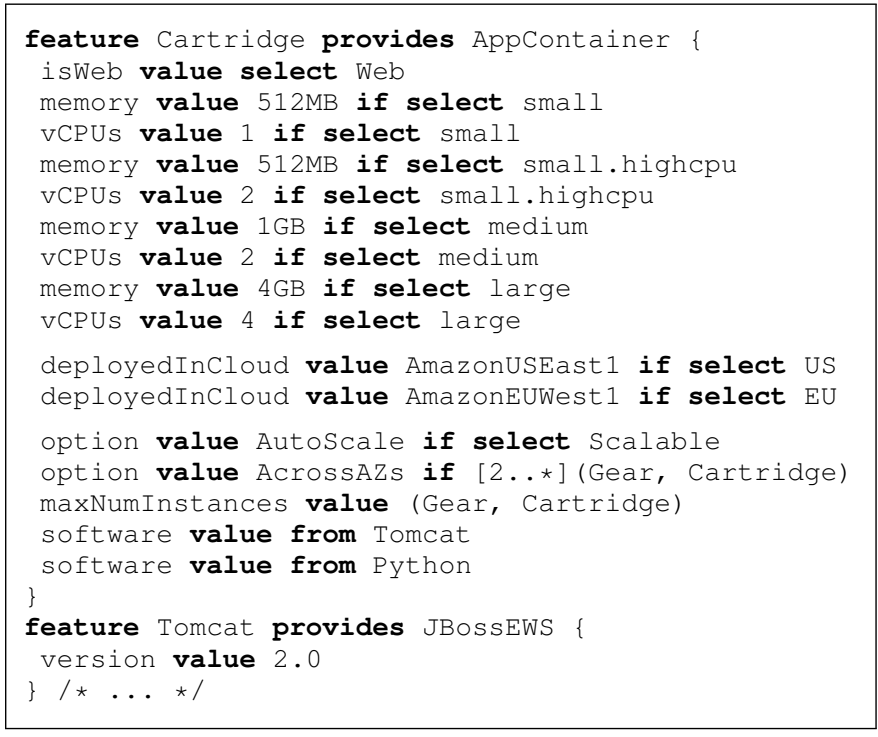

Fig. 4. Mapping from feature model for OpenShift PaaS to cloud ontology

Using ontologies has the advantage of allowing reasoning over concepts to find equivalence and specialization relationships between providers offerings and service requirements. For example, by reasoning upon ontologies we can discover that the JBoss EWS 2.0 provided by OpenShift feature Tomcat can be matched to the requirements of web frontend service (see Fig. 2), which requires a Java Servlet Container implementing version 2.5 of the Servlet API.

To enable further extensibility of our approach, application specific and provider specific ontologies can be optionally defined by developers and cloud experts respectively. Developers can define application specific concepts, as well as their relationship to global concepts, which can then be used directly in the description of the application's requirements. Similarly, provider specific concepts can be used to simplify the description of feature model mappings.

In a summary, ontologies are used to bridge the gap between service requirements and cloud providers. By using ontologies we can describe service requirements that are as complex as an ontology class description and reason upon them to find cloud providers that may offer instances of these classes.

\section{IMPLEMENTATION}

To evaluate the feasibility of our approach, we developed a set of modeling and processing tools for modeling to find a multi-cloud configuration that suits the application's requirements. The following subsections provide more information about the implementation of these tools and their evaluation.

\section{A. Modeling}

To model the artifacts required by our approach, we designed three domain-specific languages for describing (i) multi-cloud requirements; (ii) cloud provider variability; and (iii) cloud provider mappings. We then used the xText [19] framework together with Eclipse Modeling Framework [20] to

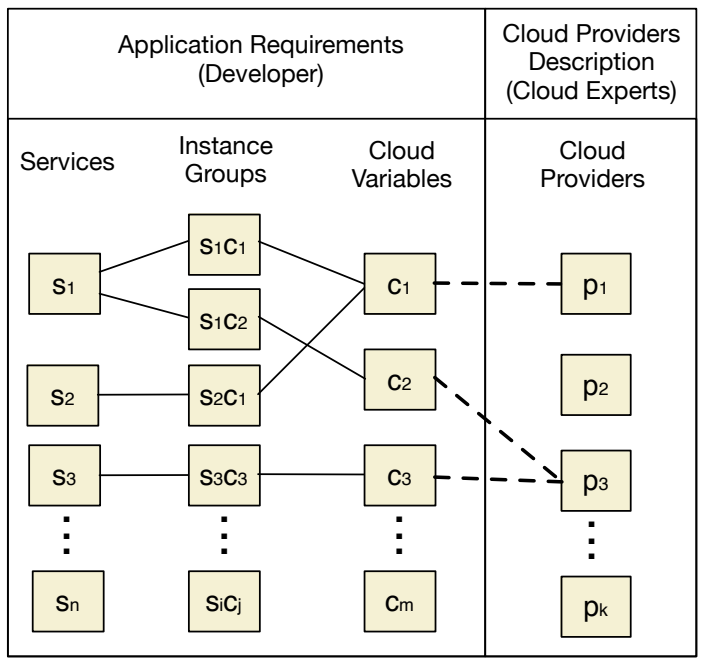

Fig. 5. Multi-cloud configuration as an assignment problem

provide an integrated editor for these languages. For describing the ontologies we use the standard OWL 2 Web Ontology Language [21], for which many modeling and reasoning tools are available [3], [22]. The developed modeling tools are available at the accompanying site 1

\section{B. Reasoning}

The problem of finding a multi-cloud configuration that suits a set of application requirements can be seen as an assignment problem subject to constraints. As illustrated in Fig. 5 an instance of this problem is described by the requirements of an application and a set of available cloud providers. From application requirements we have a set of services, which are assigned by the developer to a cloud variable through a set of instance groups (see Section III-A). On the other side, we have a set of cloud providers that offer varying functionality. A solution to an instance of this problem is an assignment from cloud variables to cloud providers, which is depicted as a set of dashed lines.

Due to the number of providers, the search space for this problem can be very large, making it difficult to find a solution in a reasonable time. In addition, verifying the suitability of a solution is a complex and time consuming task as it requires reasoning on ontologies to convert application requirements to provider specific constructs and evaluating variability in feature models. These factors, when combined, may make this problem intractable. To deal with these difficulties our search algorithm employs backtracking with constraint propagation and constraint learning [23]. We start with a set of constraints obtained from cloud conditions and increase our constraint base as verification of assignments fail. Doing so, we avoid performing expensive validations beforehand and gradually reduce the search space as new constraints are learned.

Verification is done in three steps: 1 ) validating that assigned providers comply with cloud conditions; 2) checking that

\footnotetext{
${ }^{1}$ http://researchers.lille.inria.fr/ $\sim$ sousa/mmcloud/
} 


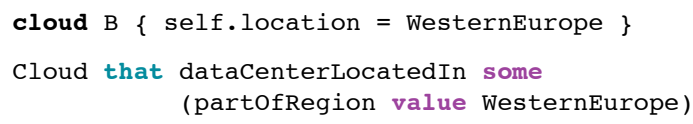

(a) Cloud variable definition and corresponding ontology class

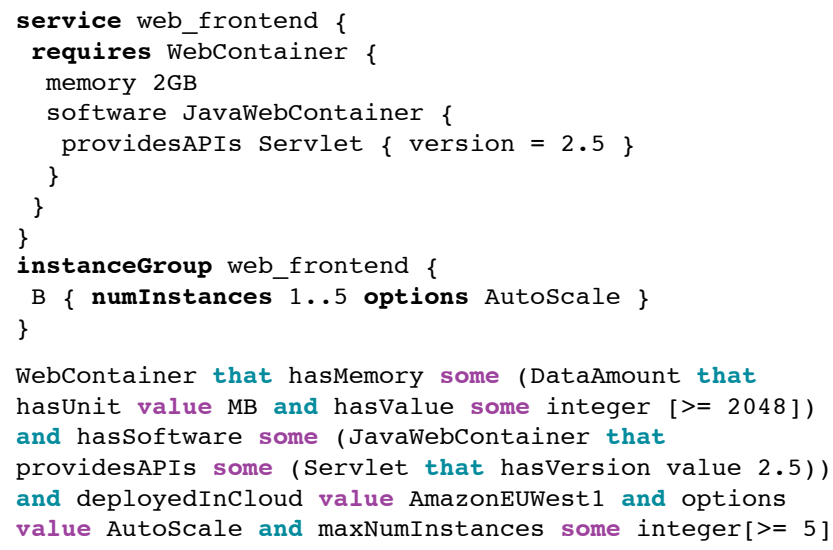

(b) Service requirements and corresponding ontology class

Cartridge \{ medium, Tomcat, EU, Scalable, 5 Gear \}

(c) Feature selection for web frontend service in OpenShift PaaS.

Fig. 6. Sample translations from requirements, to ontology classes, to features.

service requirements can be mapped to a set of features in the assigned provider; and 3) verifying that there is a valid configuration where all services assigned to a provider can be deployed. Partial results from each phase are kept in a cache to avoid repeated calculations for similar assignments.

1) Validating cloud conditions: Cloud conditions are those described in application requirements as part of the cloud variables description (see Section III-A). In Fig. 2, for example, cloud variable $B$ has as a condition that it should be assigned to a cloud located in WesternEurope. Evaluating these conditions is relatively simple and consists in checking if each assigned cloud variable complies with its constraint. However, this may still require some reasoning over ontologies to identify, for example, that a cloud located in France meets the criteria of being located in WesternEurope. To do so, we translate a cloud condition into an ontology class, which represents the class of clouds that complies with the condition, and then verify if the assigned provider cloud is an instance of this class. Fig. 6a shows an example of cloud condition for cloud variable $B$ (see Fig. 2) and the corresponding ontology class described in the OWL 2 Manchester Syntax.

2) Mapping service requirements to provider features: After verifying that the assigned providers comply with cloud conditions we still need to check if each selected provider supports all functionality required by the services assigned to it. To do so, we use the cloud provider mappings to check, for each assigned service, if its required functionality can be mapped to a set of features in the provider's feature model. First each service is converted into an ontology class, which defines the class of objects that provides service requirements. Fig. $6 \mathrm{~b}$ shows the requirements for web frontend service (see
Fig. 2) and its corresponding ontology class. Then, we use ontology reasoning together with cloud provider mappings to find a selection of features that provides an instance of this required class. Fig. 6c shows the result from mapping web frontend service to the OpenShift PaaS.

In this example, the web frontend service requires a WebContainer, but feature Cartridge in OpenShift provides just an AppContainer (see Fig. 4). However, OpenShift's specific ontology describes that a Webcontainer is the same of an AppContainer with the data property isweb set to true. OpenShift's mapping for feature Cartridge defines that the property isWeb is set to true if and only if the feature Web is also selected. Thus, an instance of feature Cartridge provides a WebContainer if its subfeature Web is selected.

The end result of this process is a feature selection defining a set of features that provide the requirements of a service. For the web frontend example, the expression obtained defines that an instance of feature Cartridge can provide service requirements if features medium, Tomcat, EU, Scalable and 5 instances of feature Gear are also selected. If a mapping from the required ontology class to a feature selection can not be found, then the assigned provider does not support the service requirements.

3) Verifying feature constraints and generating a complete configuration: After mapping service requirements to a selection of features, we know that the assigned providers support the services assigned to them. However, as there may be limits in the resources offered by a provider, conflicting features, or other constraints in cloud offerings, we still need to check if all services assigned to a provider can be deployed together and to generate a configuration for each provider. This process is done by evaluating the cloud providers' feature models against the feature selection expressions obtained in the previous step. This is a complex and time consuming task that involves translating the feature model to a constraint satisfaction problem and solving it to find a valid configuration that matches the requirements. This translation is based on existing work [24] and our previous developments [17] in cardinality-based feature models. If a solution to the corresponding constraint problem is found it means that the assignment is valid. Besides this, the output solution also defines for each selected provider a complete configuration of the corresponding feature model, detailing a hierarchy of feature instances that should be selected.

Our strategy for processing application requirements enables us to get from a set of requirements for microservices-based multi-cloud applications to a concrete set of selected features in each cloud provider. By decomposing this process into three steps we avoid doing more expensive calculations when not needed and we reuse intermediate results.

\section{Experimentation}

To validate the effectiveness of our approach and evaluate the performance of the developed tools we elaborated an example application based on the requirements described in Section [I] and measured the time consumed at each stage of 
processing, as well as the total time to get from requirements to a concrete multi-cloud configuration.

The application requirements were described using the domain-specific language for multi-cloud requirements and include 13 services, with 5 cloud variables and 15 instance groups. In addition, we elaborated feature models and ontology mappings for four popular cloud providers: OpenShif $1^{2}$ Heroku ${ }^{3}$ Google ${ }^{4}$ and Jelastic ${ }^{5}$ Information from cloud providers was obtained from their documentation and through the use of their configuration tools.

We performed 20 executions of the developed tool to convert application requirements to a multi-cloud configuration. All experiments were run on a MacBook Pro Computer with a 2 $\mathrm{GHz}$ Intel Core $\mathrm{i} 7$ processor and 8GB of memory. From the obtained results, shown in Table II we can see that translating from high-level application requirements to a concrete configuration took on average $6.62 s$. For this set of four providers 32 valid solutions, in an universe of 1024 possible assignments, were found in an average time of $24 s$.

\begin{tabular}{|l|c|c|}
\hline \multicolumn{1}{|c|}{ Operation } & $\begin{array}{c}\text { Average } \\
\text { time } \\
(\mathrm{ms})\end{array}$ & $\begin{array}{c}\text { Standard } \\
\text { deviation } \\
(\mathrm{ms})\end{array}$ \\
\hline Validate cloud conditions & 3.56 & 5.12 \\
Translate a service to feature sets & $1,796.82$ & $2,215.69$ \\
Translate a feature model to CSP and solve it & $3,350.47$ & 827.34 \\
Overall process & $6,620.60$ & $6,431.53$ \\
\hline
\end{tabular}

TABLE I

AVERAGE TIME FOR OPERATIONS IN THE GENERATION OF A MULTI-CLOUD CONFIGURATION.

To evaluate the performance in the presence of more providers we randomly generated extra 46 providers in a way that no generated provider would completely support the features required by the example application. With this new set of 50 providers, $52 s$ were need to find the same 32 solutions. This indicates, that despite the time needed to process and generate a valid solution, invalid assignments can be discarded more quickly and reduce the search space.

\section{Limitations}

Our approach focus on the selection and configuration of cloud providers for a multi-cloud scenario and does not deal directly with the deployment of applications. Generation of deployment scripts from feature models has been discussed in the literature [18], and similar techniques could be integrated in our approach. We also still do not consider costs and quality of service for optimizing the selection as these depend heavily on application usage. Finally, for the conducted experiments, cloud provider feature models and ontology mappings were manually defined relying providers' documentation, and are thus susceptible to errors.

\footnotetext{
2 http://www.openshift.com/

3 http://www.heroku.com/

${ }^{4}$ http://cloud.google.com/

5 http://jelastic.com/
}

\section{RELATED WORK}

Several recent works were proposed to deal with aspects of the management of multi-cloud systems. CloudMF [8] relies on a domain-specific language and models@runtime to deal with heterogeneity across providers and support provisioning and deployment of multi-cloud systems. We also employ domain-specific languages for modeling requirements and provider descriptions, but in addition we also support the selection of cloud providers and variability management. The mOSAIC [7] and soCloud [25] projects propose a multi-cloud PaaS for interoperability across multiple clouds. Instead of proposing developers a new platform service, our approach aims at supporting them to choose and combine existing platform, infrastructure and software services. Wright et al. [6] propose a constraints-based method for composing multiprovider environments. Like in our work, their goal is to find an assignment of services to cloud providers according to some constraints. However, requirements are defined as low-level constraints and services can use only infrastructure resources. CloudPick [9] is a framework that supports the selection of IaaS providers and the deployment of a network of virtual appliances (preconfigured virtual machine images). Cloud4SOA [26] also proposes the use of ontologies to provide interoperability across PaaS providers. Like in our approach, ontologies are used for semantically mapping requirements to providers' offerings. However, both approaches do not manage variability and constraints of cloud providers configurations.

Managing variability and heterogeneity in the cloud has also been identified as an important issue that is subject of substantial work. In [27] and [28], feature models are used to model variability in infrastructure resources (e.g. virtual machine, storage, network, etc) and support provider selection. Feature models are also used in a similar way in [29], but to optimize energy consumption. In our work, we consider not only variability in a resource or service configuration but across all the functionality offered by a provider.

Ontologies are also proposed as a way to deal with heterogeneity and the semantic gap between requirements and cloud providers offers [7], [30], [31]. However these approaches do not consider the variability in providers' offerings and apply reasoning upon predefined instances.

The SALOON [10] approach also relies on feature models to capture cloud provider configuration options and ontologies to map from requirements to cloud features. However, it does not consider applications that can be composed of multiple services and do not support multi-cloud requirements.

Our approach shares with existing work the use of ontologies to deal with heterogeneity across cloud providers and of software product line techniques for managing variability in cloud provider configurations. However, our work takes into account variability at the provider level, not only for a given resource type (virtual machine, storage, etc) and not just for one application service.

In addition, we also employ domain-specific languages and model driven-engineering techniques to achieve separation 
of concerns between different user roles. We support the description of requirements for microservices-based applications and mechanisms to achieve multi-cloud requirements of location, scalability and redundancy. Overall, our approach differentiates from others by taking into account variability in cloud configuration options, multi-cloud requirements and service composition to build a suitable environment.

\section{CONCLUSION}

Multi-cloud computing has the potential of reducing vendor dependence, increasing application reliability and optimizing resource usage. However, the wide number of available cloud providers, their high heterogeneity and their intricate configuration options, make it very complex to exploit these benefits. In this paper, we describe an approach to deal with this complexity, by supporting the selection and configuration of multicloud environments for microservices-based applications.

Our approach relies on a domain-specific modeling language for defining multi-cloud requirements and a combination of reasoning tools to obtain: (i) a valid assignment of application services to cloud providers and (ii) proper configurations for these providers. We implemented this approach as a set of tools for modeling the required artifacts and reasoning upon them. We conducted some experiments to show that valid configurations can be obtained in reasonable time.

For future work, we intend to include pricing and quality of service information as part of our analysis and investigate search algorithms and heuristics to deal with this as an optimization problem. This goes into the direction of our longterm goal, which is towards self-adaptive multi-cloud environments capable of identifying optimization opportunities as application requirements and cloud market evolve.

\section{REFERENCES}

[1] N. Grozev and R. Buyya, "Inter-cloud architectures and application brokering: taxonomy and survey," Software: Practice and Experience, vol. 44, no. 3, pp. 369-390, 2012.

[2] J. Lewis and M. Fowler. (2014, Mars) Microservices: a definition of this new architectural term. [Online]. Available: http://martinfowler. com/articles/microservices.html

[3] E. Sirin, B. Parsia, B. C. Grau et al., "Pellet: A practical OWL-DL reasoner," Web Semantics: Science, Services and Agents on the World Wide Web, vol. 5, no. 2, pp. 51-53, 2007.

[4] K. Pohl, G. Bckle, and F. J. van der Linden, Software Product Line Engineering: Foundations, Principles and Techniques, 1st ed. Springer Publishing Company, Incorporated, 2010.

[5] D. Ardagna, E. Di Nitto, G. Casale et al., "MODAClouds: A modeldriven approach for the design and execution of applications on multiple clouds," in Proc. International Workshop on Modeling in Software Engineering (MiSE '12), Zurich, Switzerland, 2012, pp. 50-56.

[6] P. Wright, Y. Sun, T. Harmer et al., "A constraints-based resource discovery model for multi-provider cloud environments," Journal of Cloud Computing: Advances, Systems and Applications, vol. 1, no. 1, p. 6, 2012.

[7] D. Petcu, B. D. Martino, S. Venticinque et al., "Experiences in building a mOSAIC of clouds," Journal of Cloud Computing, vol. 2, no. 1, pp. 1-22, 2013

[8] N. Ferry, H. Song, A. Rossini et al., "Cloud MF: Applying mde to tame the complexity of managing multi-cloud applications," in Proc. IEEE/ACM International Conference on Utility and Cloud Computing (UCC'14), London, UK, 2014, pp. 269-277.

[9] A. V. Dastjerdi, S. K. Garg, O. F. Rana et al., "CloudPick: a framework for QoS-aware and ontology-based service deployment across clouds," Software: Practice and Experience, vol. 45, no. 2, pp. 197-231, 2015.
[10] C. Quinton, D. Romero, and L. Duchien, "SALOON: a platform for selecting and configuring cloud environments," Software: Practice and Experience, vol. 46, no. 1, pp. 55-78, 2016.

[11] N. Ferry, A. Rossini, F. Chauvel et al., "Towards model-driven provisioning, deployment, monitoring, and adaptation of multi-cloud systems," in Proc. IEEE International Conference on Cloud Computing (CLOUD'13), Santa Clara, USA, June 2013, pp. 887-894.

[12] D. Petcu, E. Di Nitto, D. Ardagna et al., "Towards multi-clouds engineering," in Proc. IEEE Conference on Computer Communications Workshops, Toronto, Canada, April 2014, pp. 1-6.

[13] D. Petcu, "Multi-cloud: Expectations and current approaches," in Proc. International Workshop on Multi-cloud Applications and Federated Clouds ((MultiCloud '13), Prague, Czech Republic, 2013, pp. 1-6.

[14] L. Chen, M. Ali Babar, and N. Ali, "Variability management in software product lines: A systematic review," in Proc. International Software Product Line Conference (SPLC'09), ser. SPLC '09, San Francisco, California, USA, Aug. 2009, pp. 81-90.

[15] K. Czarnecki, S. Helsen, and U. Eisenecker, "Formalizing cardinalitybased feature models and their specialization," Software Process: Improvement and Practice, vol. 10, no. 1, pp. 7-29, 2005.

[16] C. Quinton, D. Romero, and L. Duchien, "Cardinality-based feature models with constraints: A pragmatic approach," in Proc. International Software Product Line Conference (SPLC'13), Tokyo, Japan, Aug. 2013, pp. 162-166.

[17] G. Sousa, W. Rudametkin, and L. Duchien, "Extending feature models with relative cardinalities," in Proc. International Systems and Software Product Line Conference (SPLC'16), Beijing, China, Sep. 2016.

[18] C. Quinton, D. Romero, and L. Duchien, "Automated selection and configuration of cloud environments using software product lines principles," in Proc. IEEE International Conference on Cloud Computing (CLOUD'14), Alaska, USA, Jun. 2014, pp. 144-151.

[19] L. Bettini, Implementing Domain-Specific Languages with Xtext and Xtend. Packt Publishing, 2013.

[20] D. Steinberg, F. Budinsky, E. Merks et al., EMF: Eclipse Modeling Framework. Pearson Education, 2008.

[21] S. Bechhofer, Encyclopedia of Database Systems. Boston, MA Springer US, 2009, ch. OWL: Web Ontology Language, pp. 2008-2009.

[22] B. Motik, R. Shearer, and I. Horrocks, "Hypertableau Reasoning for Description Logics," Journal of Artificial Intelligence Research, vol. 36 , pp. 165-228, 2009.

[23] R. Dechter, Constraint Processing. San Francisco, CA, USA: Morgan Kaufmann Publishers Inc., 2003.

[24] R. Mazo, C. Salinesi, D. Diaz et al., "Transforming attribute and cloneenabled feature models into constraint programs over finite domains," in Proc. International Conference on Evaluation of Novel Approaches to Software Engineering (ENASE'11), Beijing, China, Jun. 2011.

[25] F. Paraiso, P. Merle, and L. Seinturier, "soCloud: a service-oriented component-based paas for managing portability, provisioning, elasticity, and high availability across multiple clouds," Computing, pp. 1-27, 2014.

[26] E. Kamateri, N. Loutas, D. Zeginis et al., "Cloud4SOA: A semanticinteroperability paas solution for multi-cloud platform management and portability," in Proc. European Conference on Service-Oriented and Cloud Computing (ESOCC'13), K.-K. Lau, W. Lamersdorf, and E. Pimentel, Eds. , Málaga, Spain: Springer Berlin Heidelberg, Sep. 2013, pp. 64-78.

[27] A. Ferreira Leite, V. Alves, G. Nunes Rodrigues et al., "Automating resource selection and configuration in inter-clouds through a software product line method," in Proc. IEEE International Conference on Cloud Computing, (CLOUD'15), New York, United States, Jun. 2015, pp. 726733.

[28] J. García-Galán, P. Trinidad, O. F. Rana et al., "Automated configuration support for infrastructure migration to the cloud," Future Generation Computer Systems, vol. 55, pp. 200 - 212, 2016.

[29] B. Dougherty, J. White, and D. C. Schmidt, "Model-driven auto-scaling of green cloud computing infrastructure," Future Generation Computer Systems, vol. 28, no. 2, pp. 371-378, Feb. 2012.

[30] F. Amato, A. Mazzeo, V. Moscato et al., "A framework for semantic interoperability over the cloud," in Proc. of IEEE International Conference on Advanced Information Networking and Applications Workshops (WAINA'13), Barcelona, Spain, Mar. 2013, pp. 1259-1264.

[31] G. Cretella and B. Di Martino, "A semantic engine for porting applications to the cloud and among clouds," Software: Practice and Experience, vol. 45, no. 12, 2015. 